\title{
Newly Designed Ion Beam Etcher with High Etch Rate
}

\author{
Hee-Woon Cheong* \\ Department of Electrical and Computer Engineering, Seoul National University, Seoul 08826, Korea
}

(Received 17 November 2015, Received in final form 10 December 2015, Accepted 14 December 2015)

\begin{abstract}
New ion beam etcher (IBE) using a magnetized inductively coupled plasma (M-ICP) has been developed. The magnetic flux density distributions inside the upper chamber, where the plasma is generated by inductive coupling, were successfully optimized by arranging a pair of circular coils very carefully. More importantly, the proposed M-ICP IBE exhibits higher etch rate than ICP.
\end{abstract}

Keywords : IBE (Ion Beam Etcher), M-ICP (Magnetized Inductively Coupled Plasma), Plasma density, R-wave, Etch rate

\section{Introduction}

A demand for non-volatile memory devices with new characteristics such as fast access time, low operating voltage and high storage density has ever been increasing. Especially, a vibrant research over the last decade had led to the development of spin-transfer torque magnetoresistive random-access memory (STT-MRAM) [1, 2].

Patterning and etching magnetic tunnel junction (MTJ) stack in MRAM device is difficult since materials that form the stack are mostly metals which hardly form volatile etch products in plasma and are redeposited on the sidewall of the stack [3,4]. Manufacturing industries have resolved the redeposition problem by employing ion beam etcher (IBE) $[5,6]$ and performing sidewall etching. However, etch rate of MTJ stack in an IBE is too low and high ion beam energy can give severe damages to MTJ stack materials [7], which are obstacles for allowing the mass production.

In this paper, I'd like to introduce new IBE, named magnetized inductively coupled plasma (M-ICP) IBE, which exhibits high plasma density and fast etch rate.

\section{Experimental Setup}

Figure 1 shows the schematic of the IBE. The IBE consists of the upper chamber and the lower chamber.

CThe Korean Magnetics Society. All rights reserved.

*Corresponding author: Tel: +82-2-880-9554

Fax:+82-2-872-3005, e-mail: cheong1982@empas.com
Plasma is generated inside the upper chamber by inductive coupling. A two-turn planar antenna is powered by source generator which supplies $27.12 \mathrm{MHz}$ rf power. A $30 \mathrm{~mm}$-thick rf window made of quartz is employed to allow the power transfer from antenna to plasma. Meanwhile, dual-grid system is mounted on the bottom of the upper chamber to extract ion beam from the plasma. The grid system consists of the screen grid which maintains positive potential with respect to the ground and the acceleration grid which maintains negative potential with respect to the ground. The substrate holder, which is equipped with tilting and rotating modules is located inside the bottom chamber. The diameter of the substrate

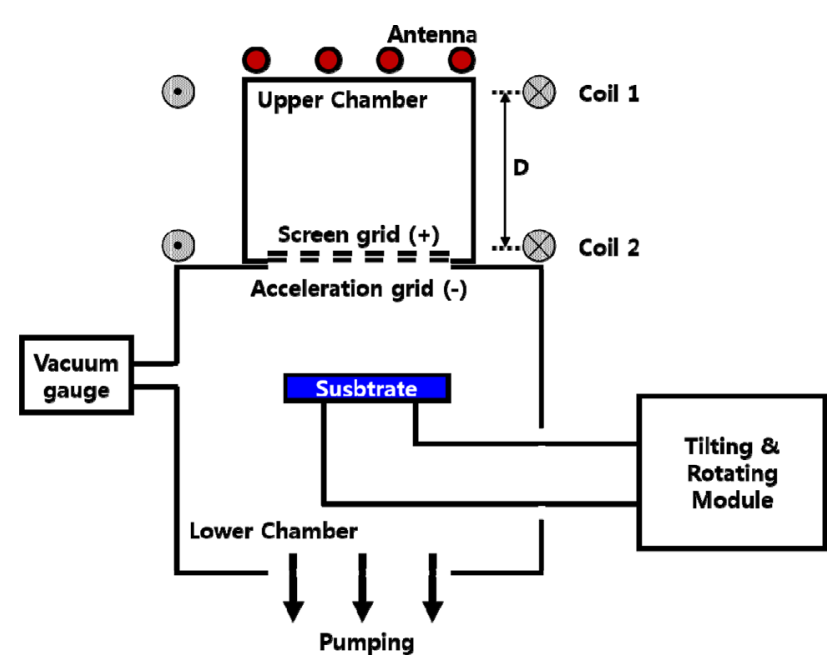

Fig. 1. (Color online) Schematic of newly designed ion beam etcher. 
holder is 4 inch and the distance between the acceleration grid and the substrate holder with its original position is $100 \mathrm{~mm}$. The IBE is pumped by a $1200 \mathrm{l} / \mathrm{s}$ turbomolecular pump and the base pressure for the whole experiment is $0.001 \mathrm{~Pa}$. Discharge gas, whose flow rate is regulated by the mass flow controller (MFC), flows into the IBE through a gas inlet and the resultant neutral gas pressure is measured by the cold cathode vacuum gauge (PfeifferVacuum IKR-251), which is located at the bottom chamber.

Meanwhile, the magnetic fields are generated inside the upper chamber using a pair of coils. The number of turns of copper wire is 1000 . The diameter of coils and the vertical distance between them must be chosen very carefully because the plasma density non-uniformity depends critically on the magnetic flux density distributions inside the upper chamber [8]. In this regard, the two-dimensional magnetic flux density distributions inside the upper chamber were simulated with the finite element method magnetic (FEMM) field calculation software to optimize the magnetic field configuration.

Plasma density was also measured in ICP IBE and MICP IBE using a dual Langmuir probe [9]. The vertical height of DLP from the screen grid was $50 \mathrm{~mm}$. More importantly, the etch characteristic of ICP IBE and M-ICP IBE was compared with line-patterned oxide sample.

\section{Results and Discussion}

Figure 2 shows the two-dimensional magnetic flux density distributions inside the chamber according to the

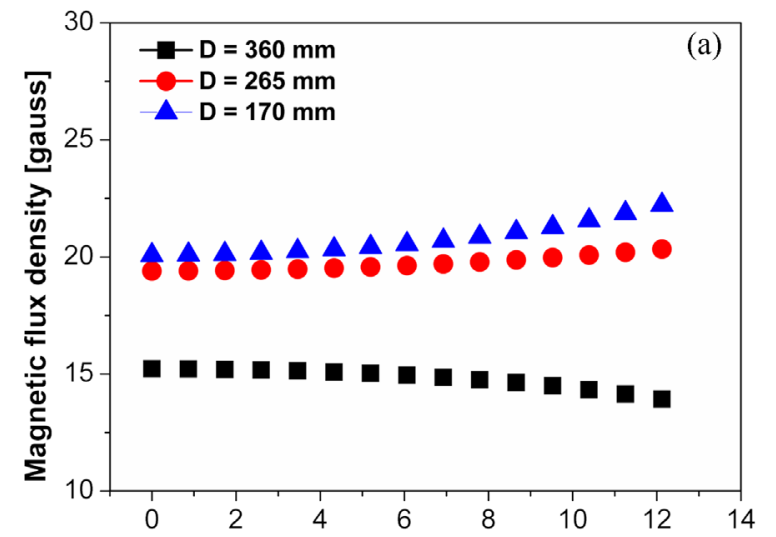

Radial distance from the center of the screen grid [cm]

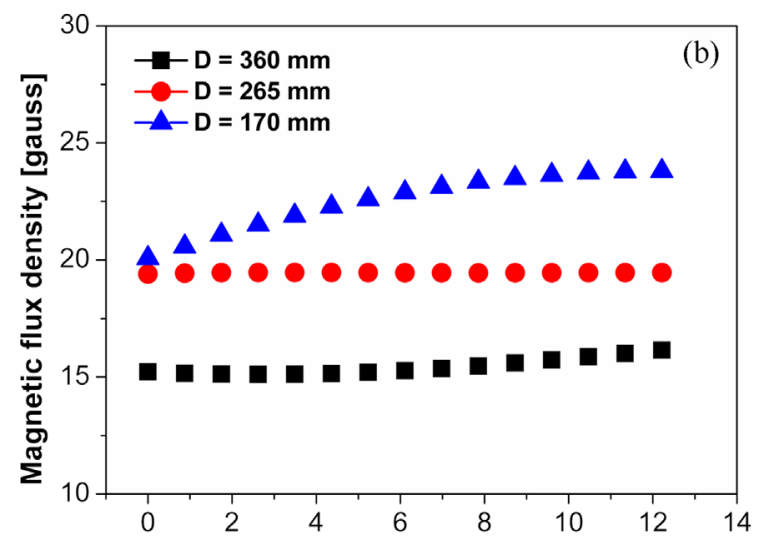

Axial distance from the center of the screen grid [cm]

Fig. 3. (Color online) Magnetic flux density according to (a) the radial distance (b) the axial distance from the center of the substrate holder.

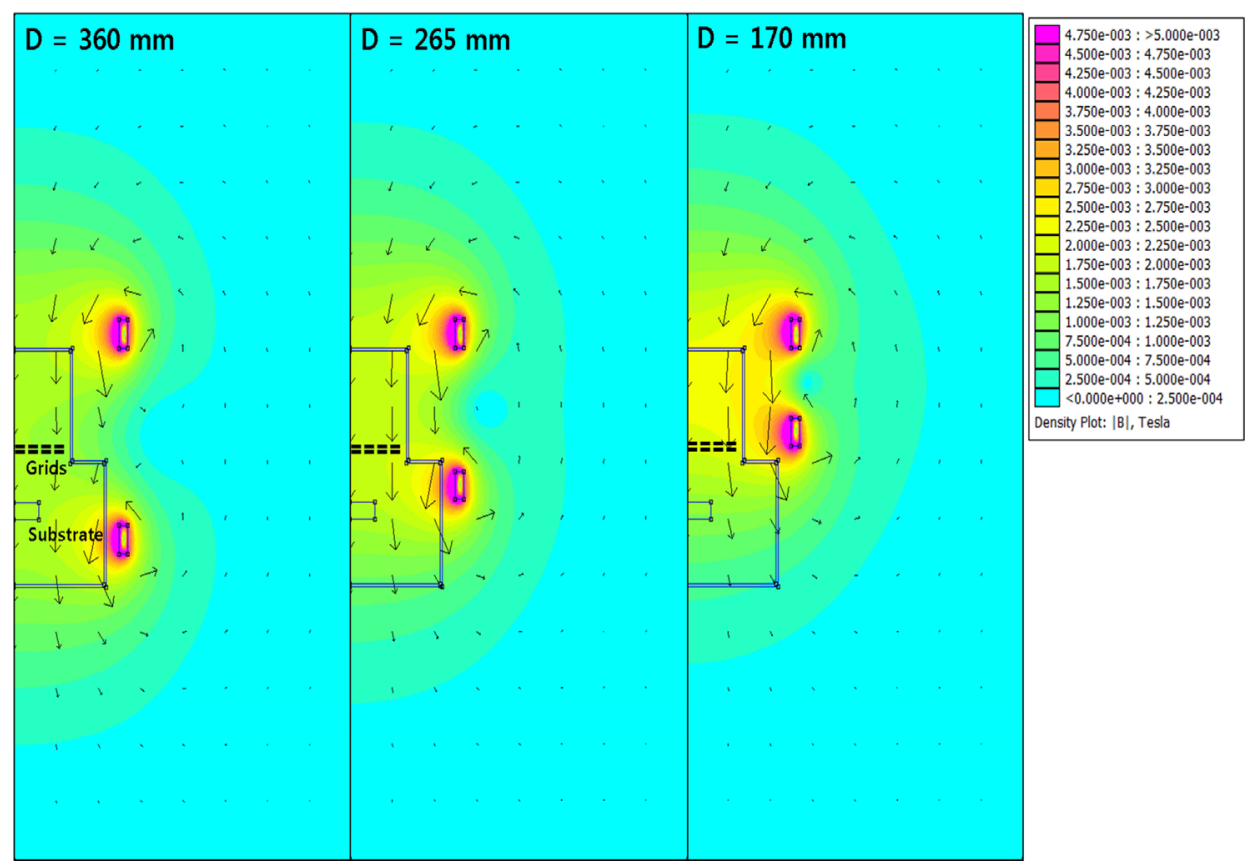

Fig. 2. (Color online) Magnetic flux density distributions according to the distance between coils. 

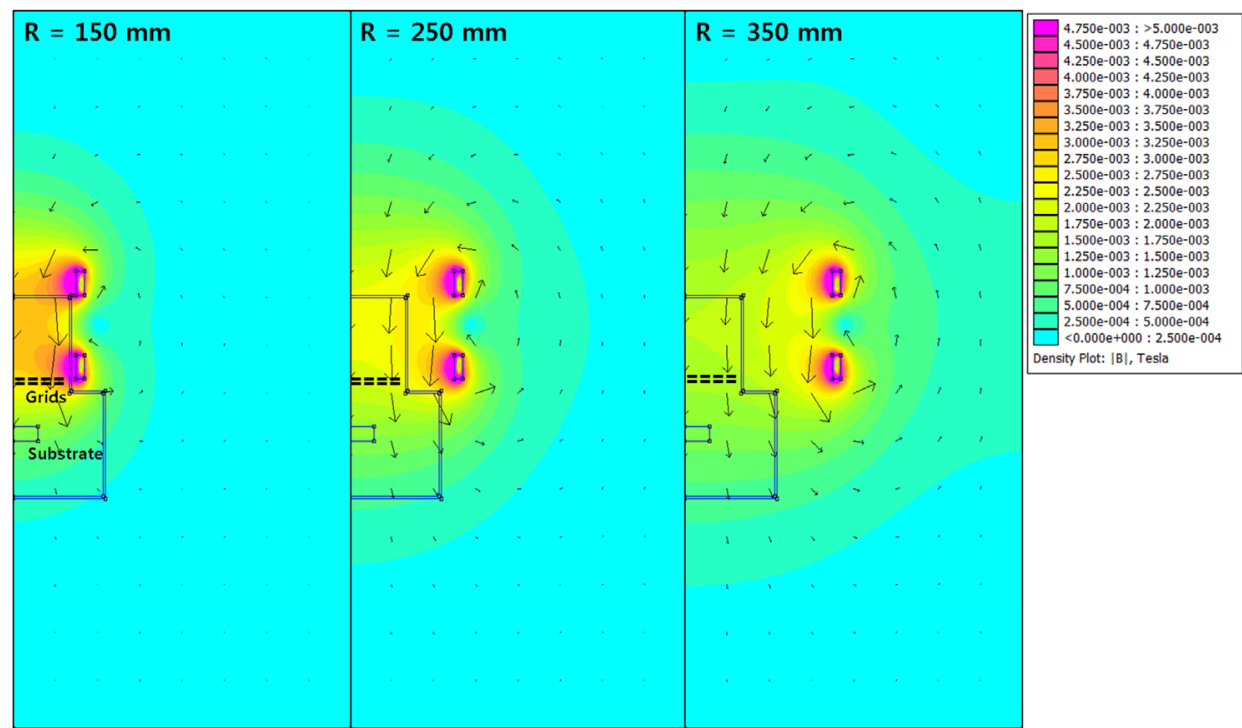

Fig. 4. (Color online) Magnetic flux density distributions according to the radius of coils.

vertical distance (D) between coils when the radius (R) of coils is $250 \mathrm{~mm}$. In the simulation, the electric current supplied to both coils was $600 \mathrm{~mA}$. Figure 3 shows radial and axial distributions of the magnetic flux density. Plasma density non-uniformity is known to be reduced when the magnetic flux density increases both radially and axially from the center of the screen grid [8]. If coils are set up symmetrically with respect to the grid system $(\mathrm{D}=360 \mathrm{~mm})$, however, the magnetic flux density decreases as the radial distance from the center of the screen grid increases, which may result in the increase in the plasma density non-uniformity due to the flute instability [8]. When two coils are placed unsymmetrically with respect to the ground system $(\mathrm{D}=265 \mathrm{~mm})$, the magnetic flux density increases as the radial distance from the center of the screen grid increases, whereas the one remains constant as the axial distance from the center of the screen grid increases. In that case, however, the dispersion relation is not satisfied and R-wave cannot be propagated into the upper chamber [10]. When the bottom coil is placed around the grid system $(D=170 \mathrm{~mm})$, on the other hand, the magnetic flux density increases as the distance from the center of the screen grid increases both radially and axially. In doing so, not only the plasma density non-uniformity can be reduced but also R-wave excitation induces electron heating.

Figure 4 shows the two-dimensional magnetic flux density distributions inside the chamber according to $\mathrm{R}$ when $\mathrm{D}=170 \mathrm{~mm}$. Figure 5 shows radial and axial distributions of the magnetic flux density. When $\mathrm{R}=150$ $\mathrm{mm}$, the magnetic flux density increases as the distance from the center of the screen grid increases both radially

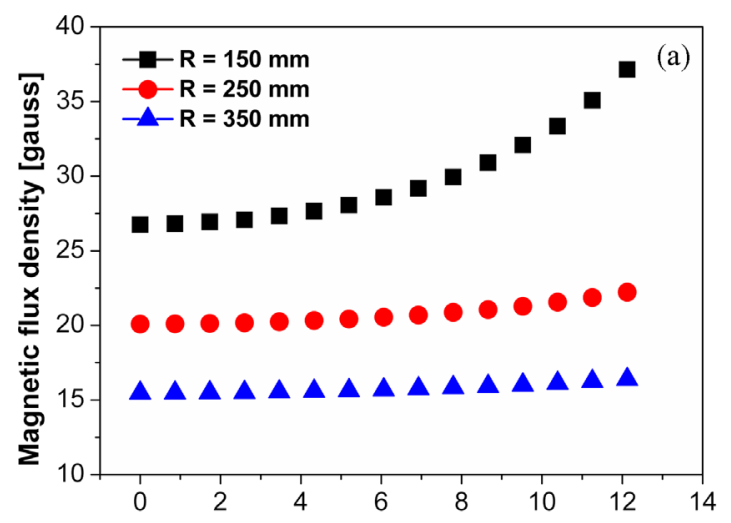

Radial distance from the center of the screen grid [cm]

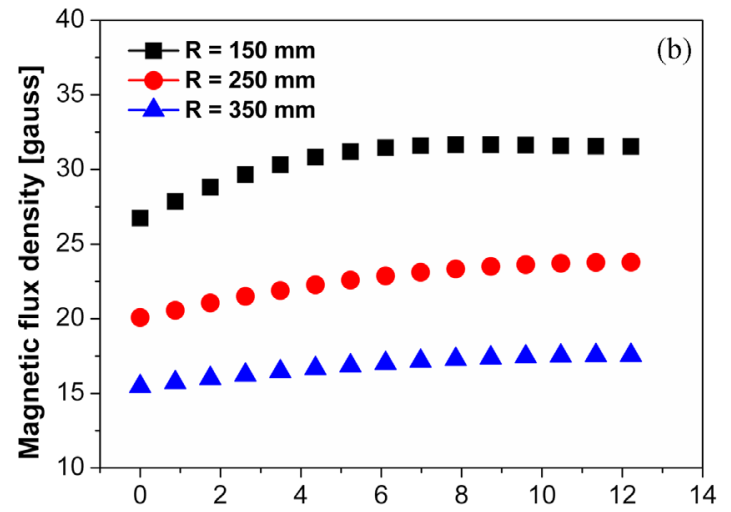

Axial distance from the center of the screen grid [cm]

Fig. 5. (Color online) Magnetic flux density according to (a) the radial distance (b) the axial distance from the center of the substrate holder.

and axially. However, the magnetic flux density vector is not perpendicular to the grid system, which causes severe 


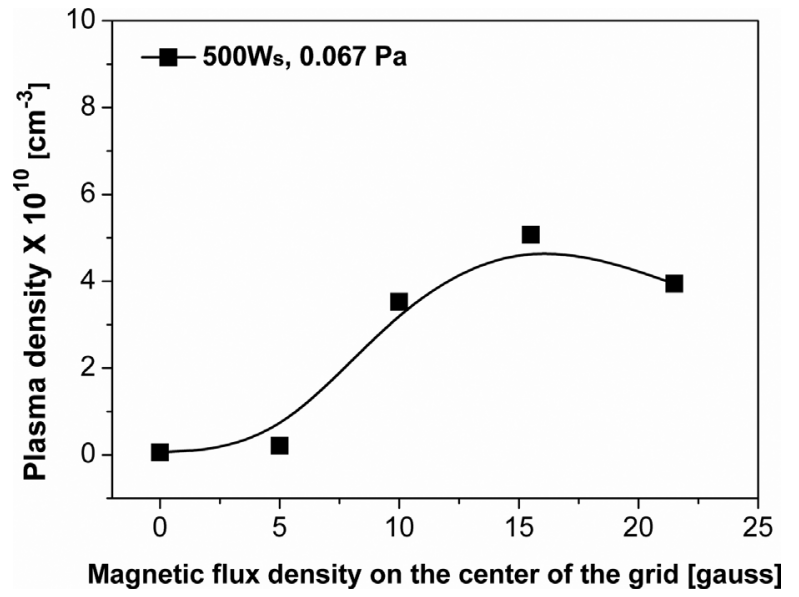

Fig. 6. Plasma density according to the magnetic flux density on the center of the screen grid.

$\boldsymbol{E} \times \boldsymbol{B}$ drift of ions [10] and the resultant distortion in ion trajectories. It is confirmed that the magnetic flux density vector is almost perpendicular to the grid system when $\mathrm{R}=350 \mathrm{~mm}$. Considering the plasma density nonuniformity, R-wave propagation and the ion trajectory, coils should be arranged very carefully. More importantly, the magnetic flux density distributions inside M-ICP IBE could be optimized properly by employing coils, the number of which is smaller than the one reported previously [8].

Figure 6 shows plasma density according to the magnetic flux density on the center of the screen grid. In this experiment, the source power was fixed at $500 \mathrm{~W}, \mathrm{Ar}$ flow rate at $3 \mathrm{sccm}$, the neutral gas pressure at $0.067 \mathrm{~Pa}$, whereas the magnetic flux density on the center of the screen grid varies from 0 gauss to 22 gauss. The plasma density of M-ICP IBE (15 gauss) is about 68.3 times higher than ICP IBE (0 gauss).

Meanwhile, the line-patterned oxide samples were prepared to compare the dry etching characteristics of ICP IBE and M-ICP IBE as depicted in Fig. 7. The photoresist (PR) is used as the mask for $\mathrm{SiO}_{2}$ patterning with a thickness of $860 \mathrm{~nm}$. The thickness of the main etching

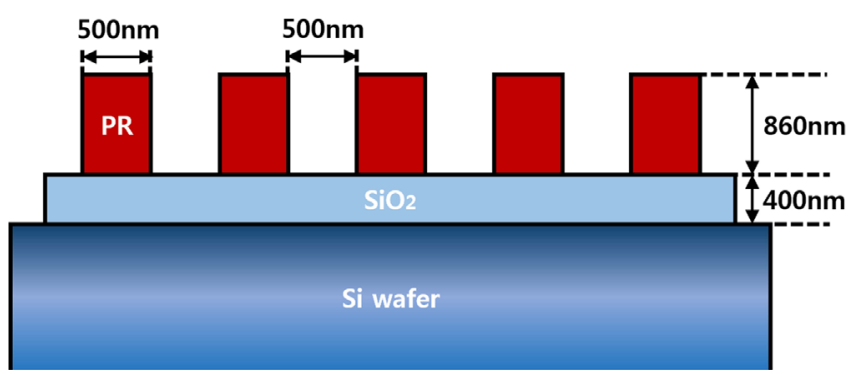

Fig. 7. (Color online) Schematic of line-patterned oxide.

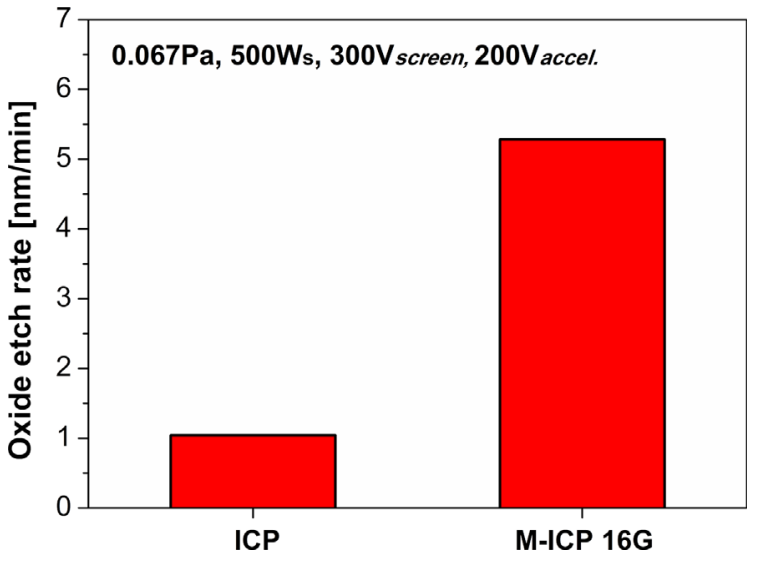

Fig. 8. (Color online) Oxide etch rate in ICP-IBE and M-ICPIBE.

layer of $\mathrm{SiO}_{2}$ is $400 \mathrm{~nm}$. The width of bar (or space) is $500 \mathrm{~nm}$.

Figure 8 shows the etch rate of oxide in ICP IBE (0 gauss) and M-ICP IBE (16 gauss). In this experiment, the source power was fixed at $500 \mathrm{~W}, \mathrm{Ar}$ flow rate at $3 \mathrm{sccm}$, the neutral gas pressure at $0.067 \mathrm{~Pa}$, the screen grid potential at $+300 \mathrm{~V}$, and the acceleration grid potential at $-200 \mathrm{~V}$. The etch time was $3 \mathrm{~min}$. M-ICP IBE shows about 5.1 times higher etch rate than ICP IBE at the fixed grid potentials due to the higher plasma density. The plasma density non-uniformity and the detailed etching mechanisms of M-ICP IBE will be reported elsewhere.

\section{Conclusions}

In this paper, newly designed M-ICP IBE was introduced. First of all, the arrangement of coils should be considered very carefully since it affects the plasma density non-uniformity, R-wave propagation, and ion beam trajectory. The magnetic flux density distributions inside MICP IBE could be optimized properly by employing two coils. Furthermore, the M-ICP IBE showed higher etch rate than ICP IBE due to its higher plasma density characteristic. Therefore, M-ICP IBE proposed in this paper could be a promising candidate for the MTJ etching.

\section{Acknowledgements}

The authors would like to thank Inter-University Semiconductor Research Center (ISRC) in Seoul National University, Seoul, Korea for full support.

\section{References}

[1] J. C. Slonczewski, J. Magn. Magn. Mater. 159, L1 (1996). 
[2] E. B. Myers, D. C. Ralph, J. A. Katine, R. N. Louie, and R. A. Buhrman, Science 285, 867 (1999).

[3] R. E. Chapman, J. Mater. Sci. 12, 1125 (1977).

[4] R. E. Lee, J. Vac. Sci. Technol. 16, 164 (1979).

[5] K. Kinoshita, H. Utsumi, K. Suemitsu, H. Hada, and T. Sugibayashi, Jpn. J. Appl. Phys. 49, 08JB02 (2010).

[6] W. J. Gallagher, S. S. P. Parkin, Yu Lu, X. P. Bian, A. Marley, K. P. Roche, R. A. Altman, S. A. Rishton, C. Jahnes, T. M. Shaw, and Gang Xiao, J. Appl. Phys. 81, 3741 (1997).
[7] K. Kinoshita, H. Utsumi, K. Suemitsu, H. Hada, and T. Sugibayashi, Jpn. J. Appl. Phys. 49, 08JB02 (2010).

[8] W. H. Lee, H. W. Cheong, J. W. Kim, and K. W. Whang, Plasma sources Sci. Technol. 24, 065012 (2015).

[9] Y. Sung, H. B. Lim, and R. S. Houk, J. Anal. At. Spectrom 17, 565 (2002).

[10] H. W. Cheong, "Design of a Magnetized Inductively Coupled Plasma Reactor for Large Area Plasma and a Study on Its Process Characteristics", Ph.D. dissertation Seoul National University (2014). 